\title{
Numerical Study of the Anisotropic Properties of Vortex Motion in Superconducting Films with a Periodic Lattice of Deffects
}

\author{
I. G. R. Pitta, Clécio C. de Souza Silva, and J. Albino Aguiar \\ Departamento de Física, Universidade Federal de Pernambuco, \\ 50670-901 Recife, PE, Brasil
}

Received on 28 February, 2002

\begin{abstract}
The anisotropic effects on the dynamical phases of a vortex lattice driven by a uniform Lorentz force are investigated by numerical simulations. A square array of columnar defects is represented by a periodic pinning potential. We assume that flow is elastic, so that the vortex-vortex interactions can be represented by elastic forces. V-I characteristics and mean-square displacements and velocities for different orientations of the driving force are calculated. It is found that, for a wide range of driving force magnitude and orientation, the vortex lattice is transversely pinned in high symmetry directions of the pinning potential. In addition, the transverse pinned vortex lattice is thermally depinned at high enough temperatures.
\end{abstract}

\section{Introduction}

The transport properties in a type-II superconductor are dominated by the quantized flux lines, vortices, which are present in the sample in a wide range of current and magnetic field. In these samples, electrical resistivity is zero as long as there is no colective motion of vortices. As a transport current is applied, this is only possible if the pinning force produced by material defects or artificial pinning centers is strong enough to counter-balance the Lorentz force produced by the current. In addition to, thermal fluctuations should be sufficiently small to prevent thermally ativated flux motion through the pinning barriers. If these conditions are not satisfied, the vortex lattice (VL) starts moving and competition between the VL internal forces and interaction with the effective pinning potential can lead, depending on the current strength, to different dynamics which determine the voltage-current (V-I) characteristics.

Lattice structures driven by an external force over a periodic or disordered substrate have attracted growing interest. These systems usually exhibit a variety of complex phenomena and dynamical phases that can dominate transport properties. Physical systems in which dynamical phases play an important role include boundary lubrication [1], charge density waves, Wigner cristals, Josephson junction arrays (JJA) [2] and vortex lattices [3-5]. The development of several techniques to artificially fabricate periodic structures in superconducting samples [6] and the recent advances in real time vortex imaging make driven vortex assemblies interacting with periodic pinning a specially usefull system to probe theories on dynamical phases and phase transitions.

In this work, we use a simple elastic model to study the dynamics of vortex lattices driven by an external force in a superconducting film with periodic array of columnar defects. Individual vortex motion is described by overdamped Langevin equations which account for first-neighbors elastic interactions, square pinning potential and uniform driving force $\mathbf{F}_{d}$. The transport properties are determined by calculating the timeaveraged VL center of mass (CM) velocity as a function of $\mathbf{F}_{d}$. We find that the V-I curves are anisotropic and transverse pinning of the moving VL in high symmetry directions of the square pinning array is observed. We also study the stability of this phase with respect to thermal fluctuations. We find that the transversely pinned VL can be thermally depinned above certain temperatures. These results are in agreement to those obtained by more realistic computer simmulations $[3,4]$ and to recent experiments in [5].

\section{Numerical details}

We simulated the dynamics of a vortex lattice in thin superconducting films with a square lattice of columnar deffects. We consider a two dimensional square 
lattice of vortices interacting with their first neighbors by elastic forces, i. e., $\mathbf{F}_{v v}=-\kappa \mathbf{d}$, where $\mathbf{d}$ is the distance between two nearby vortices and $\kappa$ is the elastic coefficient. For this preliminar study, we used a small system, namely $8 \times 8$ vortices, with a concentration of one vortex per deffect, and periodic boundary conditions were considered. The defect lattice is modeled by a periodic pinning potential. To simplify our study we expand the pinning potential in a Fourier series, $U^{v-p}(\mathbf{r})=\sum_{\mathbf{Q}} U_{\mathbf{Q}} e^{i \mathbf{Q} \cdot \mathbf{r}}$, where $\mathbf{Q}$ are reciprocal lattice vectors of the square pinning lattice, considering only the high symmetry directions of the square lattice, $[1,0]$, $[0,1]$ and $[1,1]$, that is, the Fourier components of the pinning potential are given by:

$$
U_{\mathbf{Q}}= \begin{cases}U_{1} & \mathbf{Q}= \pm \frac{2 \pi}{a_{p}} \hat{x}, \pm \frac{2 \pi}{a_{p}} \hat{y} \\ U_{2} & \mathbf{Q}= \pm \frac{2 \pi}{a_{p}}(\hat{x} \pm \hat{y}) \\ 0 & \text { otherwise }\end{cases}
$$

Here we assume $U_{1}=0.1$ and $U_{2}=U_{1} / 2$. This gives a square potential as shown in Fig. 1.

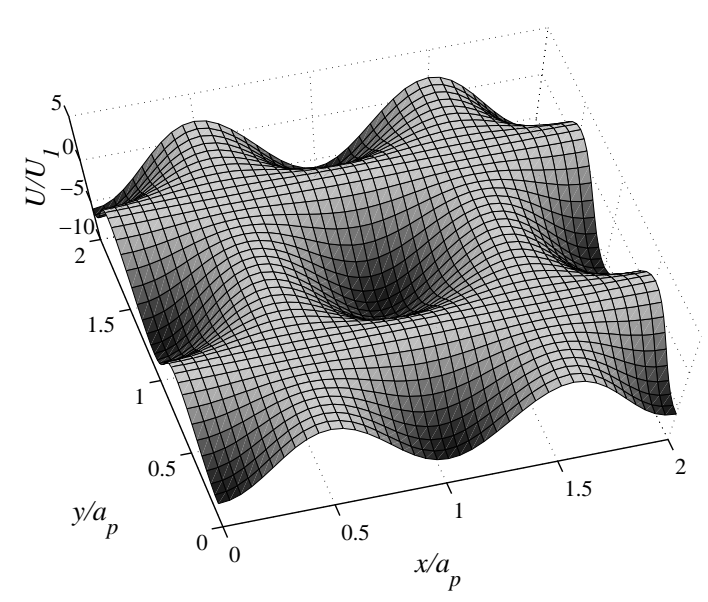

Figure 1. Surface plot of the square pinning potential given by Eq. (1) and using $U_{2}=U_{1} / 2$.

Thermal fluctuations are added by applying an appropriated Brownian force $\boldsymbol{\Gamma}_{i}$ in each vortex. This force is a gaussian, stochastic variable with zero average and represents the thermal noise, being related to the temperature by the fluctuation-disspation theorem.

The driving force $\mathbf{F}_{d}$ is the Lorentz force due to an externally applied current $\mathbf{J}$, i.e., $\mathbf{F}_{d}=\phi_{0} \hat{\nu} \cdot \mathbf{J}$, where $\nu$ is the unit vector parallel to the vortex symmetry axis. Approximating vortices to massless particles, vortex dynamics can be described by overdamped equations of motion,

$$
\eta \mathbf{v}_{i}=\mathbf{F}_{v v}-\nabla U_{p}+\mathbf{F}_{d}+\boldsymbol{\Gamma}_{i}
$$

where $\eta$ is the frictional coefficient. From now on, $\kappa, \eta$ and the potential periodicity $a_{p}$ are taken to be units.

\section{Results and discussion}

For small enough driving forces, the vortex lattice interactions with the pinning centers do not allow the lattice to flow. By increasing the driving force slightly, depinning occurs and the vortex lattice starts flowing. In this initial flow the vortex motion is disordered or plastic (Fig. 2-a) and reorder into a lattice (Fig. 2-b) as $\mathbf{F}_{d}$ increases. This reordering effect is expected, since, for high enough driving forces, the pinning potential as felt by the moving vortex assembly is avareged out in the direction of motion. For vortex motion in some high symmetry axis, the moving VL interacts with a static "washboard" potential periodic in the direction perpendicular to $\mathrm{CM}$ motion and the ordered vortex lattice can be either commensurate or incommensurate with this washbord potential, depending on the pinning strength. For motion in other directions the average pinning potential desapears completely. This leads to strongly anisotropic transport properties.
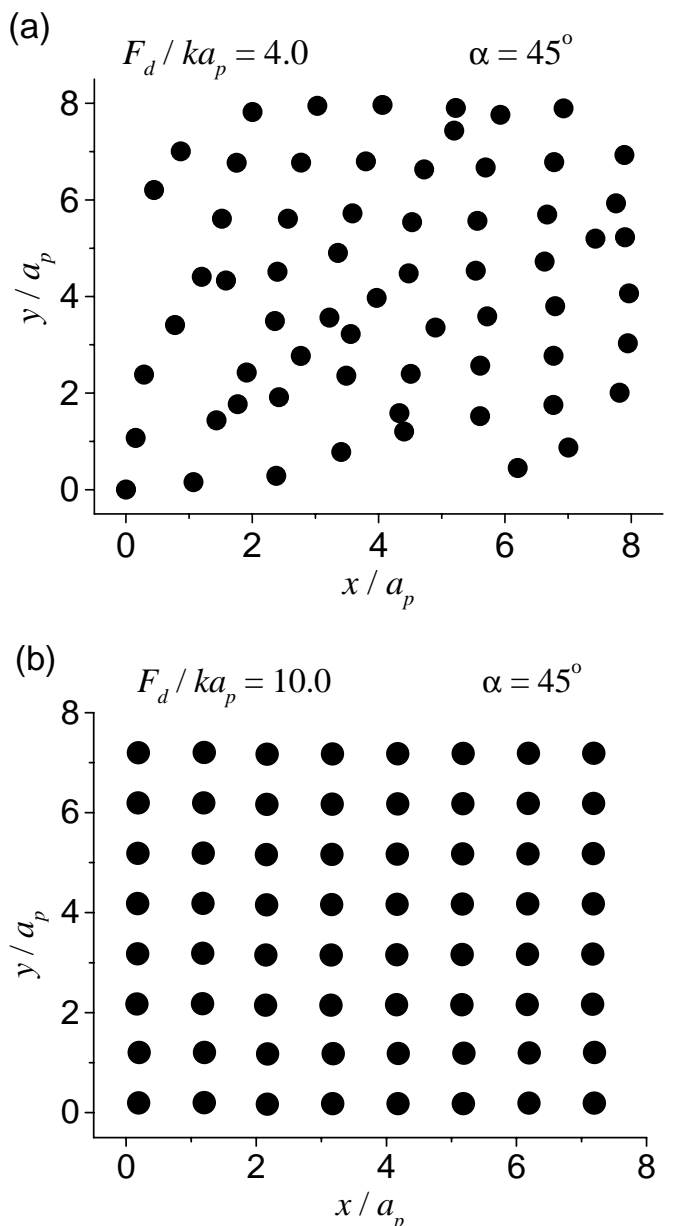

Figure 2. Snapshots of the vortex assembly for (a) $F_{d} / \kappa a_{p}=$ 4.0 (disordered flow) and (b) $F_{d} / \kappa a_{p}=10.0$ (ordered lattice flow).

To study this anisotropic properties, we used in our simulations $\mathbf{F}_{d}$ directions $\theta$, with respect to the $x$ axis, 
ranging from 0 to $45^{\circ}$ in multiples of $5^{\circ}$. In Figs. 3-a and 3-b $\mathbf{F}_{d}$ is applied at zero temperature and the horizontal $\left(v_{x}\right)$ and vertical $\left(v_{y}\right)$ components of VL center of mass velocities are calculated. As can be seen in Fig. 3-a the onset of vortex motion in $\mathrm{x}$ and $\mathrm{y}$ directions occurs for different $\mathbf{F}_{d}$ intensities. For $\mathbf{F}_{d}$ orientations between 0 and $25^{\circ}$, after the depinning threshold, the motion remains trapped (or transverselly pinned) in $[1,0]$ direction until $F_{y}$ reaches a critical value. As seen in Fig. 3-b, for higher angles $\left(0^{\circ}<\theta<45^{\circ}\right)$, the VL is pinned in the $[1,1]$ direction $\left(v_{x}=v_{y}\right)$. In Figs. 3 -c and 3 -d we made the same simulation using nonzero temperatures. This figure shows that the critical transverse depinning force decreases as temperature increases, which means that the pinning potential effects are softened by thermal fluctuations.

To study in further details the transverse depinning, $\mathbf{F}_{d}$ was initially fixed in the horizontal direction $\left(F_{x}=4.0 / \kappa a_{p}\right)$ and $F_{y}$ raised from $F_{y}=0$ up to 5.5 $\kappa a_{p}$ for four different temperatures: from $k_{b} T=0$ up to $k_{b} T=0.06 \kappa a_{p}^{2}$, as shown in Fig. 4. The range of forces where the VL motion remains in the transversely pinned phase - directions $[1,0]$ and $[1,1]$ - decreases as temperature increases. In this way, it is possible to transversely depin the VL by raising the temperature. This is illustrated by Fig. 5, where the temperature dependence of transverse depinning is shown for fixed $F_{x}=4.0 \kappa a_{p}$ and for three different $F_{y}$ intensities, namely: $F_{y}=1.05 \kappa a_{p}, F_{y}=1.15 \kappa a_{p}$ and $F_{y}=1.25 \kappa a_{p}$. A $T-F_{y}$ dynamical phase diagram, for $F_{x}$ fixed at $4.0 / \kappa a_{p}$, is shown in Fig. 6. Three phases can be distinguished: two transversely pinned phases, one in the $[1,0]$ direction and another in the $[1,1]$ direction, and a floating solid phase, where no trapping occur.

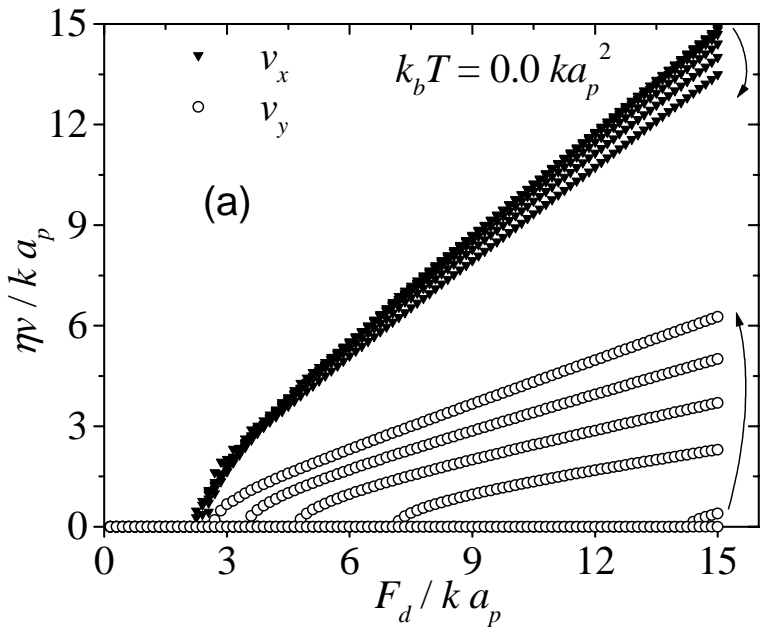

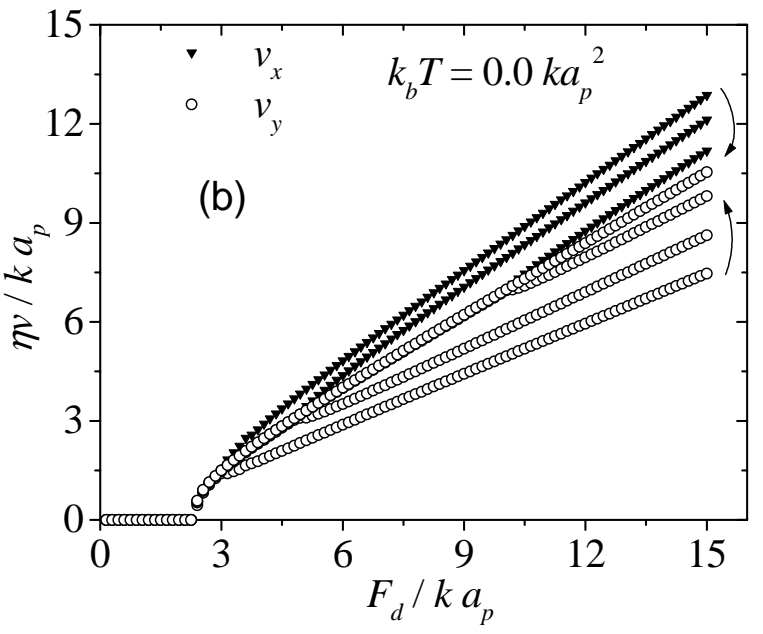
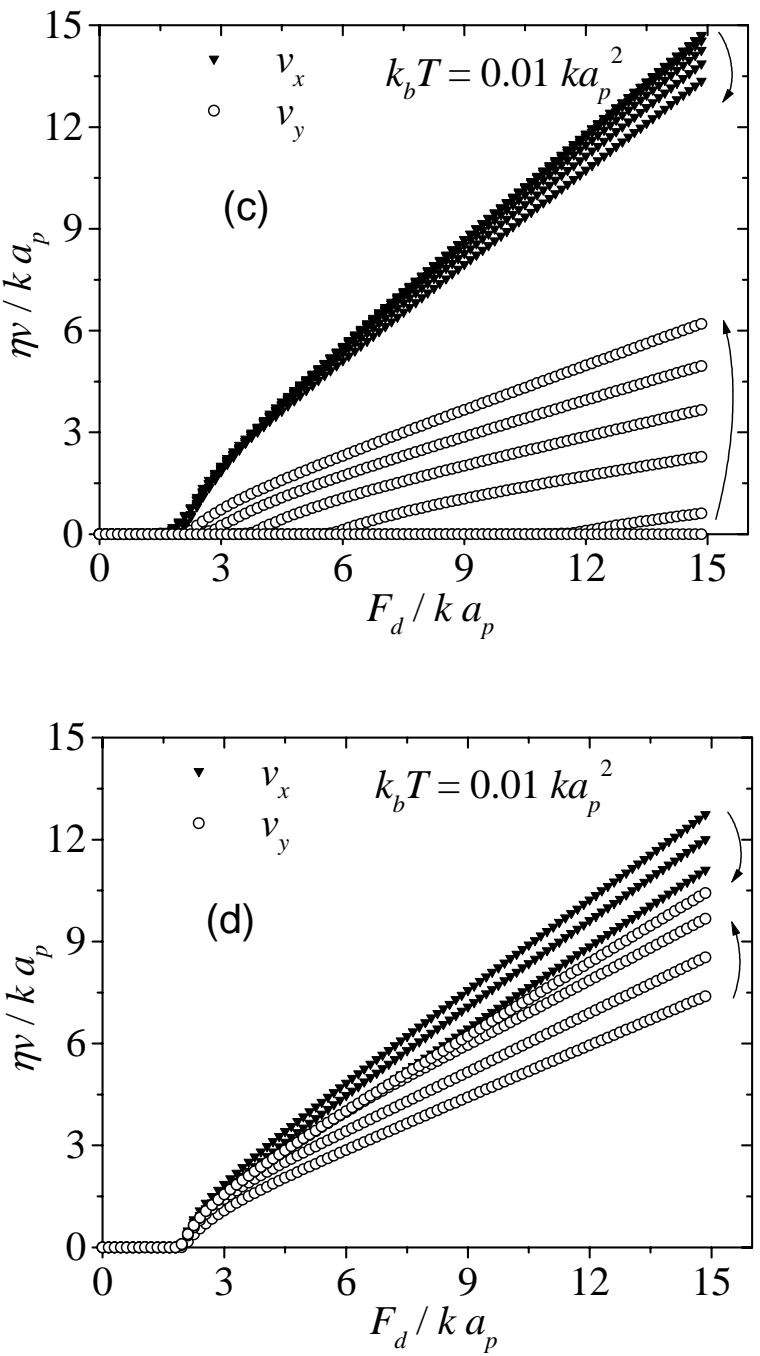

Figure 3. Vortex lattice velocity as a function of the driving force for $\theta$ ranging, in multiples of $5^{\circ}$, from 0 to $25^{\circ}$, (a) and (c), and from $30^{\circ}$ to $45^{\circ}$, (b) and (d). The arrows indicate increasing $\theta$. (a), (b) are at zero temperature and (c), (d) at $k_{b} T=0.01 \kappa a_{p}^{2}$. 


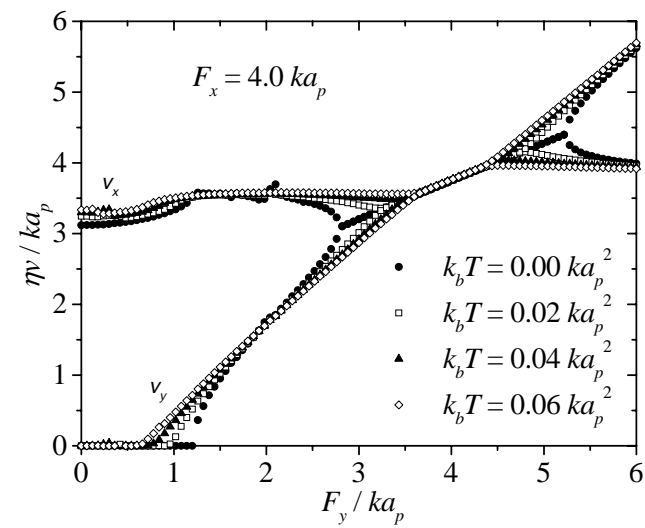

Figure 4. $v_{x}$ and $v_{y}$ as functions of $F_{y}$ for $F_{x}$ fixed at $4.0 \kappa a_{p}$ and different temperatures.

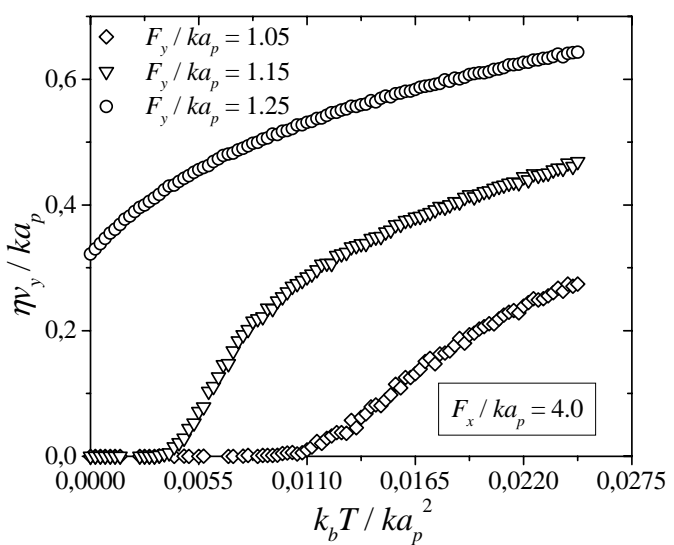

Figure 5. Temperature dependence of the transverse depinning with $F_{x}$ fixed at $4.0 \kappa a_{p}$ and three different intensities of $F_{y}$.

\section{Conclusion}

In conclusion we have numerically simmulated a vortex lattice in a superconducting film with a square array of pinning centers driven by a uniform driving force. As a simplification, the vortices were considered to interact only with their first neighbors via elastic forces. The results agree qualitatively with recent experiments [5] and numerical simulation of long-range interacting vortices [3] and JJA [4]. The V-I characteristics, represented by the CM velocity vs. driving force curves, have shown to be highly anisotropic, and it was observed transverse pinning in the directions $[1,0]$ and $[1,1]$ of the square pinning lattice. We have also studied the temperature dependence of the transverse CM velocity which illustrate thermal transverse depinning. Our results on transverse pinning are summarized in the dynamical phase diagram of Fig. 6, where the onset of transverse voltage are plotted.

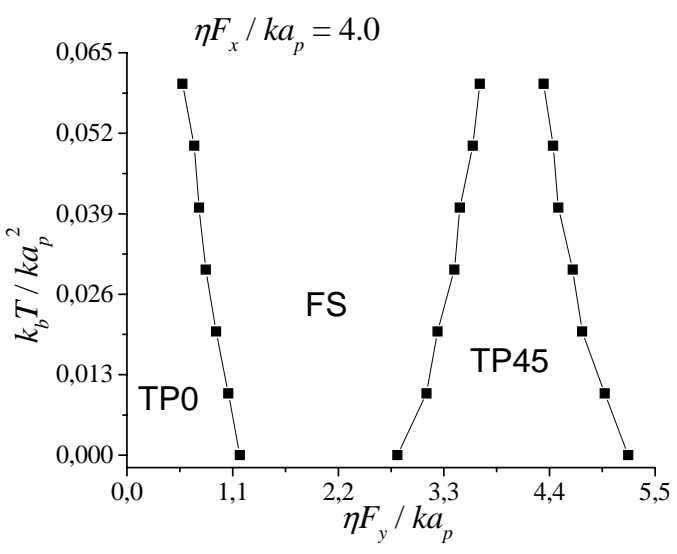

Figure 6. Dynamical phase diagram; showing three phases: trasnversaly pinned in directions $[1,0]$ (TP0) and $[1,1]$ (TP45) and a depinned or floating solid phase (FS).

\section{Acknowledgments}

This work was sponsored by the Brazilian Agencies CNPq and FACEPE. We thank L.R.E. Cabral for usefull suggestions and stimulating discussions.

\section{References}

[1] B.N.J. Persson, Sliding Friction: Physical Principles and applications (Springer, Heidelberger, 1998).

[2] R. S. Newrock, C.J. Lobb, U. Geigenmller, and M. Octavio, Solid State Phys. 54, 263 (2000).

[3] G. Carneiro J. Low Temp. Phys. 117, 1323 (1999); Phys. Rev. B 62, 14661 (2000).

[4] V.I. Marconi, S. Candia, P. Balenzuela, H. Pastoriza, D. Dominguez, Phys. Rev. B 62, 4096 (2000).

[5] V.I. Marconi and D. Dominguez, Phys. Rev. Lett. 82, 4922 (1999); Phys. Rev. B 63, 174509 (2001).

[6] A.N. Lykov, Adv. Phys. 42, 263 (1993). V.V. Moshchalkov, V. Bruyndoncx, L. Van Look, M.J. Van Bael, Y. Bruynseraede, and T. Tomomura, Handbook of Nanostructured Materials and Nanotechnology, Vol. 3, H.S. Nalwa ed. (Academic Press, 2000). 